\title{
Riqueza y abundancia de mamíferos en un ambiente antropizado en Zongolica, Veracruz
}

\section{Richness and abundance of mammals in an antropized environment in Zongolica, Veracruz}

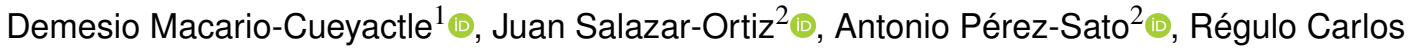 \\ Llarena-Hernández ${ }^{1}$ (D), Nancy María Alavéz-Martínez ${ }^{1}$, Ricardo Serna-Lagunes ${ }^{1 *}$ [D] \\ ${ }^{1}$ Facultad de Ciencias Biológicas y Agropecuarias, región Orizaba-Córdoba, Universidad Veracruzana. Josefa Ortiz de Domíngez s/n \\ Peñuela CP. 94945. Amatlán de Los Reyes, Veracruz, México. \\ ${ }^{2}$ Colegio de Postgraduados, Campus Córdoba. Congregación Manuel León s/n, Amatlán de los Reyes, Veracruz, México. \\ *Autor de correspondencia: rserna@uv.mx
}

Artículo científico recibido: 19 de diciembre de 2018 aceptado: 01 de agosto de 2019

RESUMEN. Los patrones de diversidad de mamíferos suelen ser afectados por pérdida del hábitat y presencia humana. En Veracruz se tiene un inventario de 195 mamíferos, pero en áreas geográficas aisladas como la región Altas Montañas, se desconoce la dinámica de la diversidad de mamíferos. El objetivo del estudio fue evaluar la riqueza y abundancia de mamíferos en dos vertientes del Cerro Acontecatl, Zongolica, Veracruz, México. Se utilizó la técnica de fototrampeo y se establecieron transectos para identificar rastros de las especies de mamíferos a partir de 12 muestreos durante mayo a septiembre de 2014. Con un total de $7704 \mathrm{~h}_{\text {trampa }}^{-1}$ y $144000 \mathrm{~m}^{2}$ muestreados, se registraron 11 mamíferos pertenecientes a siete familias: Didelphidae, Sciuridae, Cuniculidae, Mephitidae, Procyonidae, Cervidae y Felidae. Leopardus wiedii y $L$. pardalis, dos felinos registrados en este estudio se encuentran en peligro de extinción. El índice de Shannon mostró similitud en las dos vertientes del Cerro Acontecatl, mientras que el índice de Margalef indica un ecosistema medianamente diverso en cuando a riqueza y abundancia de mamíferos. La curva de acumulación de especies indicó que se debieron registrar 12 especies con una cobertura de muestreo del 91\%. En el área de estudio se detectaron actividades de caza, por este motivo el Cerro Acontecatl debe declararse como un Área Prioritaria para la Conservación, donde se fomente la educación ambiental en la ciudad de Zongolica para concientizar sobre la importancia ecológica de los mamíferos que habitan la zona periurbana de la cabera municipal.

Palabras clave: Área Prioritaria para la Conservación, bosque fragmentado, cacería, mastofauna.

ABSTRACT. Mammalian diversity patterns are usually affected by habitat loss and human presence. In Veracruz there is an inventory of 195 mammals, but in isolated geographical areas such as the Altas Montañas region, the dynamics of mammal diversity are unknown. The objective of the study was to evaluate the richness and abundance of mammals in two slopes of Cerro Acontecatl, Zongolica, Veracruz, Mexico. The phototramp technique was used and transects were established to identify traces of mammalian species from 12 samples during May to September 2014. With a total of 7704 $\mathrm{h} / \mathrm{trap}$ and 144,000 $\mathrm{m}^{2}$ sampled, 11 mammals belonging to seven families: Didelphidae, Sciuridae, Cuniculidae, Mephitidae, Procyonidae, Cervidae and Felidae. Leopardus wiedii and L. pardalis, two felines registered in this study are in danger of extinction. The Shannon index showed similarity in the two slopes of Cerro Acontecatl, while the Margalef index indicates a moderately diverse ecosystem in terms of mammal wealth and abundance. The species accumulation curve indicated that 12 species had to be registered with a sampling coverage of $91 \%$. Hunting activities were detected in the study area, for this reason Cerro Acontecatl must be declared as a Priority Area for Conservation, where environmental education is encouraged in the city of Zongolica to raise awareness about the ecological importance of the mammals that inhabit the peri-urban area of the municipal capital.

Key words: Priority Conservation Area, fragmented forest, hunting, mastofauna. 


\section{INTRODUCCIÓN}

México cuenta con 550 especies de mamíferos distribuidos en 201 géneros, 46 familias y 13 órdenes; roedores y murciélagos son los más diversos, contribuyendo con más del $70 \%$ de las especies de mamíferos, seguido por los carnívoros, cetáceos, insectívoros y lagomorfos (Ceballos y Arroyo-Cabrales 2012). Esta diversidad posiciona a México en uno de los países con mayor riqueza de mamíferos (Ceballos y Brown 1995). Sin embargo, los problemas ambientales en México han contribuido a que ocho especies se hayan extinguido (Ceballos et al. 2002), en tanto que 238 especies (40\%) enfrentan problemas de conservación (Ceballos y Oliva 2005). Para el estado de Veracruz se reporta una riqueza de 195 especies de mamíferos entre los que destaca el orden Carnivora (22 especies), Rodentia (55 especies), Lagomorpha (5 especies) y una especie del orden Cingulata (González-Christen y Delfín-Alfonso 2016), de las cuales 53 están protegidas por la NOM-059SEMARNAT-2010 (SEMARNAT 2010), pero poco se conoce sobre la cantidad de especies que viven o pueden vivir con éxito en diferentes regiones del estado (González-Christen 2010, González-Christen y Delfín-Alfonso 2016).

Debido a su sensibilidad a las alteraciones del hábitat, principalmente aquellas causadas por actividades antrópicas, al respecto se sabe que los mamíferos se consideran indicadores del estado de conservación de los ecosistemas en los que se distribuyen (Ramírez-Pulido et al. 2005). Este taxón representa un papel importante en el ecosistema, ya que se consideran especies sombrilla de vertebrados e invertebrados, ya que protegen a otras especies asociadas al hábitat; tienen un rol preponderante en el ecosistema al ser dispersores de semillas, depredadores, polinizadores y participan en la cadena alimenticia en los eslabones de depredadores y presas (Stoner et al. 2007). Los estados de Oaxaca, Chiapas y Veracruz albergan la mayor diversidad de mastofauna en México, en particular Veracruz es considerado el tercer estado más diverso en mastofauna, pero es alarmante el número de especies incluidas en alguna categoría de riesgo de extinción en la NOM059-SEMARNAT-2010 y en el libro rojo de especies en riesgo de Veracruz (Hernández-Baz y RodríguezVargas 2014). A esta importancia y problemática de la mastofauna de Veracruz, se suma la carencia de estudios actualizados, inventarios biológicos y el estudio de poblaciones mastozoológicas, los cuales son necesarios para entender, los patrones de distribución y abundancia de mamíferos silvestres (González-Christen 2011), información básica para emprender acciones de manejo y protección (Gaona et al. 2003).

La región centro del estado de Veracruz se caracteriza por presentar los últimos remanentes de bosque mesófilo de montaña (BMM, García-Franco et al. 2008). En cafetales aledaños a estos bosques, la cacería ilegal de mamíferos no es circunstancial y responde al uso extractivo de este recurso alimenticio por las comunidades humanas que habitan a los alrededores del BMM. Una amenaza constante en las poblaciones de mamíferos, es que la intensidad de caza se incrementa en zonas con mayor densidad de población humana (Tlapaya y Gallina 2010). Los mamíferos en BMM exigen un hábitat particular en cuanto a alimento, refugio, protección y son un grupo clave en el mantenimiento de este ecosistema (García-Burgos et al. 2014). Los ecosistemas de la zona montañosa del centro de Veracruz y en particular el BMM del municipio de Zongolica se encuentran fragmentados por diferentes actividades antropogénicas (CONABIO 2011); en adición, es una zona poco explorada y estudiada en cuanto a la diversidad de mamíferos con presencia en la región (Hernández-Huerta 1994). Por tal motivo, se requieren inventarios y estudios sobre la dinámica de comunidad de mamíferos y sus amenazas, que sirvan de sustento en la elaboración de estrategias de conservación del taxón y su hábitat (González-Ruiz et al. 2014). Por las razones expuestas anteriormente, el objetivo del estudio fue estimar la riqueza y abundancia de mamíferos en el Cerro Acontecatl, Zongolica, Veracruz, para tener información básica que apoye las estrategias de conservación. 


\section{MATERIALES Y MÉTODOS}

\section{Área de estudio}

El presente estudio se realizó en el Cerro Acontecatl (1 $879 \mathrm{msnm}$ ), ubicado a $2 \mathrm{~km}$ de la cabecera municipal de Zongolica, Veracruz; colinda con las comunidades de Tenango, Acontla y Tonalixco Chico. El tipo de vegetación presente en la zona de estudio es BMM, con clima semicálido y templado húmedo con neblina y frecuentes lluvias.

La zona de estudio se dividió en dos áreas con la finalidad de realizar la comparación en la diversidad de mamíferos: la vertiente Oeste y vertiente Este (Figura 1). La primera es considerada una zona más perturbada, ya que se encuentra cercana a la cabecera municipal con accesos (veredas) que facilitan el paso a los humanos; lo que incrementa la facilidad de realizar actividades agropecuarias y otras actividades clandestinas como la tala de árboles, recolecta de orquídeas, extracción de suelo para su venta como abono, cacería, entre otras actividades. La segunda es una zona más conservada en cuanto a la vegetación presente, con cobertura vegetal superior al $80 \%$ en el estrato arbóreo, 70 y $60 \%$ en el estrato arbustivo y herbáceo, respectivamente, con especies vegetales representativas de BMM, donde se observa menor perturbación y grado de intervención humana.

\section{Técnicas de muestreo}

Se realizó un recorrido prospectivo al área de estudio para identificar y describir las características fisiográficas y para ubicar el área adecuada donde se instalarían las fototrampas y para trazar los transectos. De mayo a septiembre del 2014 se efectuaron 12 muestreos, cada uno con duración de dos días, con diferencia de 15 días en promedio entre muestreos. Se establecieron 12 transectos, seis en la vertiente Oeste y seis en la Este de $1000 \mathrm{~m}^{2}$ (500 $\mathrm{m}$ de longitud por $2 \mathrm{~m}$ de ancho); en cada transecto se empleó la técnica de recorridos por transecto de franja (Mandujano 2011), buscando rastros (huellas, pelos y heces) de mamíferos que fueron identificados mediante las claves de Aranda $(2000,2012)$. El esfuerzo de muestreo de los transectos se cal- culó multiplicando el número de transectos recorridos por el número de veces recorridos $\left(12000 \mathrm{~m}^{2} \times 12\right.$ muestreos) de acuerdo con Mandujano (2011).

De marzo a septiembre de 2014 se colocaron cuatro fototrampas, dos en la vertiente Oeste y dos en la vertiente Este. La colocación se realizó con base en las sugerencias de Chávez et al. (2013). Las cámaras se programaron para trabajar las $24 \mathrm{~h}$ del día, registrando 3 disparos cada $5 \mathrm{~s}$ al detectar movimiento. Cada una de las fototrampas fue rotada en tres diferentes estaciones, con lo que se obtuvo un total de 12 estaciones de muestreo (Figura 1). Las fotocapturas se seccionaron como registros independientes de cada especie, considerando individuos diferentes a: 1) animales en fotografías consecutivas de la misma especie plenamente distinguibles, 2) en fotografías con una separación de 3 $h$ en las que no fue posible identificar a cada ejemplar de la misma especie y 3 ) a cada individuo en fotografías de múltiples individuos (Ávila-Nájera et al. 2016). El esfuerzo de muestreo de las cámaras trampa se calculó multiplicando el número de cámaras por días de monitoreo (Gallina y GonzálezRomero 2018).

Se diseñó y aplicó una entrevista a 10 habitantes del área de estudio. Los criterios de selección de los entrevistados fueron: personas adultas mayores de 50 años que residen en la zona de estudio, campesinos con conocimientos empíricos sobre los procesos naturales y que hayan experimentado al menos alguna vez actividades de cacería (Tirira 1998). A los que se les mostraron una serie fotográfica de mamíferos con distribución en la región reportados en la literatura (Ceballos et al. 2005, González-Christen 2006, 2011, Aranda 2012 y CONABIO 2011), para luego preguntarles si en algún momento cazaron y/o observaron a la especie de la fotografía. Con el objetivo de realizar una comparación de los registros del estudio con los registros históricos identificados en literatura y entrevistas, para conocer las especies que se han perdido de la región.

\section{Análisis de datos}

Se elaboró un listado taxonómico de los 


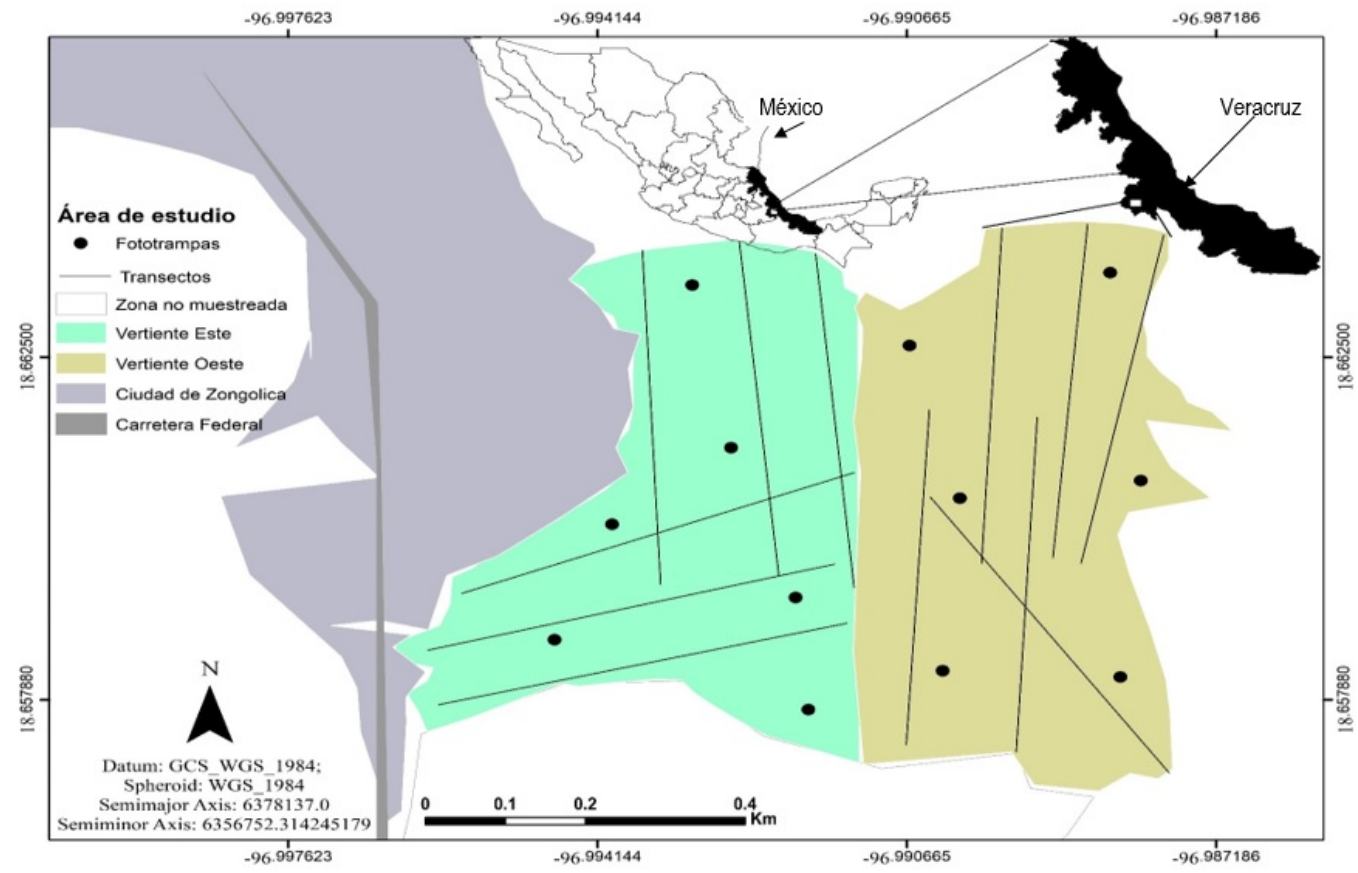

Figura 1. Área de estudio en el Cerro de Acontencatl. El cerro se dividió en dos zonas de muestreo, Vertiente Este y Oeste. Se muestran los transectos (líneas en negro), las fototrampas (puntos negros) y las zonas aledañas al área de estudio.

mamíferos registrados mediante el fototrampeo y transectos, usando la nomenclatura y clasificación de Wilson y Reeder $(2005,2011)$, actualizada con base en Ramírez-Pulido et al. (2014). El estatus de conservación de cada especie se constató en la NOM059-SEMARNAT-2010 (SEMARNAT 2010).

Los datos obtenidos en campo se capturaron en una hoja de cálculo de Excelß con información sobre la especie, la forma de obtención del registro y la vertiente en la que se registró. La riqueza regional (diversidad gamma) se consideró como el número total de mamíferos del Cerro Acontecatl. Para las especies con registros independientes en el fototrampeo, la abundancia de cada especie se cuantificó como el número de registros totales de cada especie entre el número total de registros de todas las especies multiplicado por 100, para presentarlo en porcentaje (Moreno et al. 2011). Para conocer la representatividad de mamíferos en el período de muestreo, se obtuvo la frecuencia relativa por ocurrencia (FRO) para cada especie (Altamirano-Álvarez et al. 2009, Lavariega et al. 2012).
Con el software EstimateS 8.2 (Colwell 2013) se obtuvo el índice de Margalef (DMG), el cual, cuando toma valores menores de 2 , de 2 a 3.5 y mayores de 3.5 se considera baja, media y alta diversidad de especies y abundancias, respectivamente (Margalef 1972). Para probar que ambas vertientes muestreadas son iguales, se utilizó el índice de equidad de Shannon, y para fortalecer la comparación, se obtuvo el índice de Jaccard $(\mathrm{J})$, ya que este índice se usa para estimar la diversidad compartida (Moreno 2001). Para evaluar la eficiencia del muestreo se calcularon las curvas de acumulación de especies en cada vertiente con el paquete iNEXT (Chao et al. 2016), usando inter y extrapolaciones del orden $q$ (diversidad alfa); los intervalos de confianza se calcularon al $95 \%$ a partir de 100 remuestreos por bootstrap; para obtener la salida gráfica que estima la completitud del muestreo con base a la riqueza y número de individuos registrados y estimar las especies posibles que se debieron registrar (Colwell 2006, Chao y Jost 2012). 


\section{RESULTADOS}

Con un total de $7704 \mathrm{~h} \mathrm{trampa}^{-1}$ y 144000 $\mathrm{m}^{2}$ muestreados, se registró la diversidad gamma (riqueza regional) con un total de 11 especies con las dos técnicas de muestreo. Con la técnica del fototrampeo se registraron 10 mamíferos: Didelphis marsupialis, Didelphis virginiana, Sciurus aureogaster, Cuniculus paca, Mazama temama, Nasua narica, Procyon lotor, Conepatus leuconotus, Leopardus wiedii y Leopardus pardalis. Dos especies se registraron por transectos: Philander opossum y $M$. temama, la primera mediante visualización directa y la segunda por huellas. Estas especies se agrupan en cuatro órdenes, siete familias y ocho géneros. De las 16 especies de mamíferos que se registraron mediante revisión de literatura, $12(75 \%)$ especies se corroboraron por entrevistas (Tabla 1).

De las 11 especies registradas, L. wiedii y L. pardalis se encuentran se encuentran en Peligro de Extinción (P) con bajas abundancias (Figura 2). Cinco especies presentaron abundancias superiores al $12 \%$ que en su conjunto suman más del $95.6 \%$ de la abundancia de todas las especies, donde $D$. marsupialis fue la especie más abundante $(29 \%)$ en cuanto al número de registros; seguida de $N$. narica con $23 \%$ (Figura 2). Por otro lado, seis especies presentaron abundancias bajas menores al $3 \%$, que en su conjunto representan el $4.4 \%$ de las abundancias registradas en el muestreo (Figura 3). Para la FRO, $D$. marsupialis se encontró en el $100 \%$ de los muestreos; $D$. virginiana, $N$. narica, $S$. aureogaster, $M$. temama, C. paca y $C$. leuconotus presentaron entre 83 y $50 \%$ de FRO; mientas que L. wiedii, P. lotor y P. opposum tuvieron la menor frecuencia con un registro (Figura 4).

En la vertiente Oeste se registraron 9 especies, mientras que en la vertiente Este fue de 8 especies. De acuerdo con índice de Margalef $\left(\mathrm{D}_{M G}=2.1\right)$, el Cerro Acontecatl presenta una diversidad mediana en riqueza y abundancia de mamíferos. En la comparación del índice de diversidad de Shannon entre vertientes, los resultados no mostraron diferencias significativas entre la diversidad y abundancia de mamíferos en ambas vertientes, ya que los interva- los de confianza al $95 \%$ se traslapan (Figura 5), esto debido a que los sitios comparten siete especies: $D$. marsupialis, D. virginiana, $N$. narica, M. temama, $S$. aureogaster, C. paca y C. leuconotus, lo cual fue consistente con los valores del índice de Jaccard ( $\mathrm{J}$ $=0.7$ ). Por su parte, la curva de acumulación de especies en cada vertiente no fue significativamente diferente, ya que los intervalos de confianza se sobreponen; a pesar del muestreo realizado, se debieron haber registrado al menos 12 especies, es decir, se tuvo una completitud de muestreo del 91\% (Figura 6).

\section{DISCUSIÓN}

La región de las Altas Montañas del estado de Veracruz, donde se realizó el presente trabajo, se encuentra en la zona de transición de la región biogeográfica Neártica y Neotropical; caracterizada por su riqueza mastofaunística (Ceballos et al. 2002). Las especies reportadas en este trabajo son una primera aproximación para incrementar el conocimiento de la distribución actual de los mamíferos medianos y grandes de la sierra de Zongolica, destacando la presencia de $L$. weidi y $L$. pardalis de los seis felinos que se distribuyen en México, lo que es un indicador de una simpatría en el uso de recursos por este grupo de carnívoros (CruzJácome et al. 2015). Las dos especies de felinos registrados se encuentran en riesgo de extinción, por lo que se debe conservar su hábitat (Ceballos y Brown 1995); ya que estas especies requieren de amplias masas de bosque para cubrir sus requerimientos de nicho (Torres-Romero et al. 2017). Por lo que conectar los fragmentos del paisaje de la región con las áreas naturales protegidas como el Parque $\mathrm{Na}$ cional Pico de Orizaba y Parque Nacional Cañón del Río Blanco podrían contribuir a la conservación de estas y otras especies asociadas.

Las 27 especies de mamíferos registrados por las distintas técnicas de muestreo empleadas representan el $12 \%$ de las 222 especies de mamíferos terrestres reportadas para México (Ceballos y Oliva 2005) y el 14\% de las 195 especies de mamíferos reportadas para Veracruz (GonzálezChristen y Delfín-Alfonso 2016). Porque la zona estu- 
Macario-Cueyactle et al.

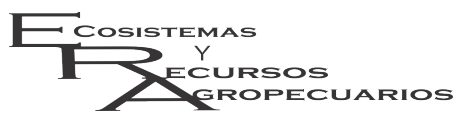

Mamíferos en ambientes antrópicos

Ecosist. Recur. Agropec. 6(18):411-422,2019

Tabla 1. Lista de especies con registros históricos en la región de las Altas Montañas, Veracruz. Registros obtenidos a partir de listado potencial y su confirmación por entrevistas.

\begin{tabular}{cccc}
\hline No. & Especies con distribución histórica en la región & Registradas en la literatura & Confirmadas por entrevistas \\
\hline 1 & Glaucomys volans (Linnaeus, 1758) & $\mathrm{X}$ & $\mathrm{X}$ \\
2 & Mustela frenata (Lichtenstein, 1831) & $\mathrm{X}$ & $\mathrm{X}$ \\
3 & Caluromys derbianus (Waterhouse, 1841) & $\mathrm{X}$ & $\mathrm{X}$ \\
4 & Potos flavus (Lichtenstein, 1832) & $\mathrm{X}$ & $\mathrm{X}$ \\
5 & Dasypus novemcinctus (Linnaeus, 1758) & $\mathrm{X}$ & $\mathrm{X}$ \\
6 & Tamandua mexicana (Saussure, 1860) & $\mathrm{X}$ & $\mathrm{X}$ \\
7 & Odocoileus virginianus (Zimmermann, 1780) & $\mathrm{X}$ & $\mathrm{X}$ \\
8 & Tayassu tajacu (Linnaeus, 1758) & $\mathrm{X}$ & $\mathrm{X}$ \\
9 & Sphiggurus mexicanus (Kerr, 1792) & $\mathrm{X}$ & $\mathrm{X}$ \\
10 & Dasyprocta mexicana (Saussure, 1860) & $\mathrm{X}$ & $\mathrm{X}$ \\
11 & Bassariscus astutus (Lichtenstein, 1830) & $\mathrm{X}$ & $\mathrm{X}$ \\
12 & Urocyon cinereoargenteus (Schreber, 1775) & $\mathrm{X}$ & \\
13 & Lynx rufus (Schreber, 1777) & $\mathrm{X}$ & $\mathrm{X}$ \\
14 & Herpailurus yagouaroundi (Geoffroy Saint-Hilaire, 1803) & $\mathrm{X}$ & \\
15 & Puma concolor (Linnaeus, 1758) & & $\mathrm{X}$ \\
16 & Panthera onca (Linnaeus, 1758) & & \\
\hline
\end{tabular}

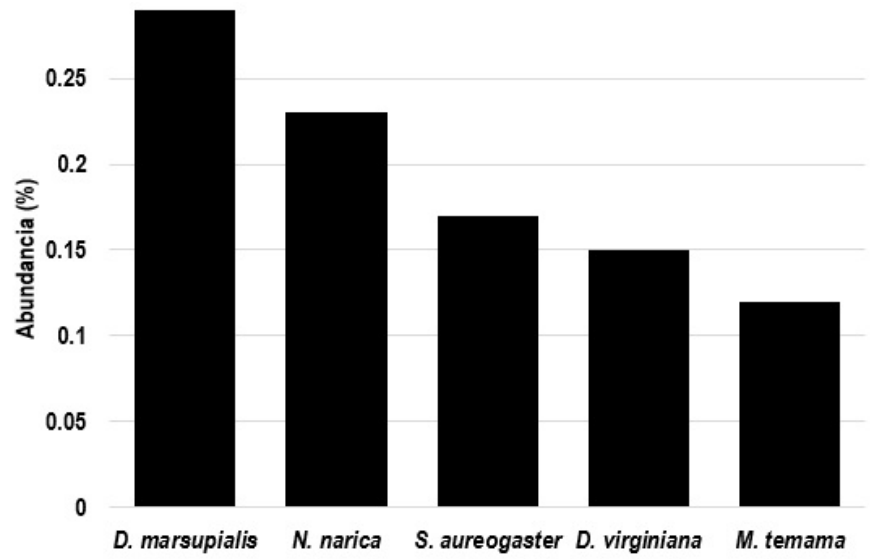

Figura 2. Abundancia de mamíferos del cerro Acontecatl, Zongolica, Veracruz. Las cinco especies en su conjunto reúnen más del $95.4 \%$ de la abundancia total registrada.

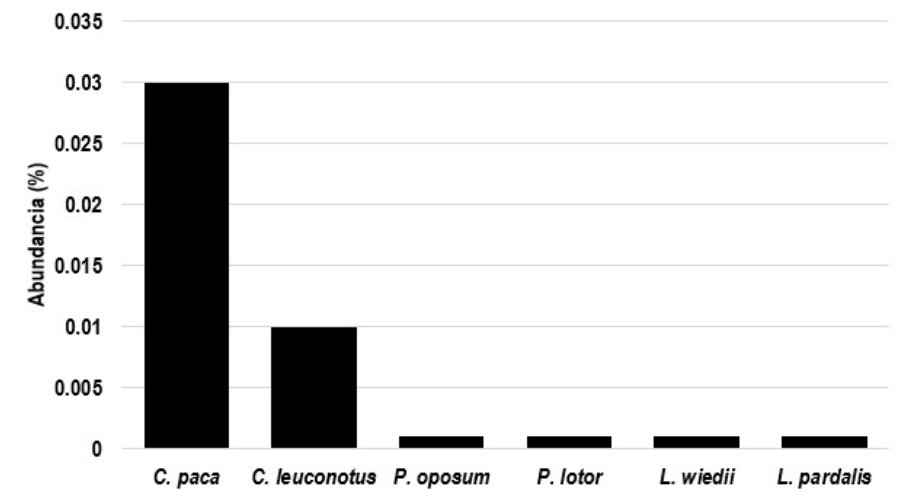

Figura 3. Abundancia de mamíferos del cerro Acontecatl, Zongolica, Veracruz. Las seis especies en su conjunto reúnen el $4.6 \%$ de la abundancia total registrada. 


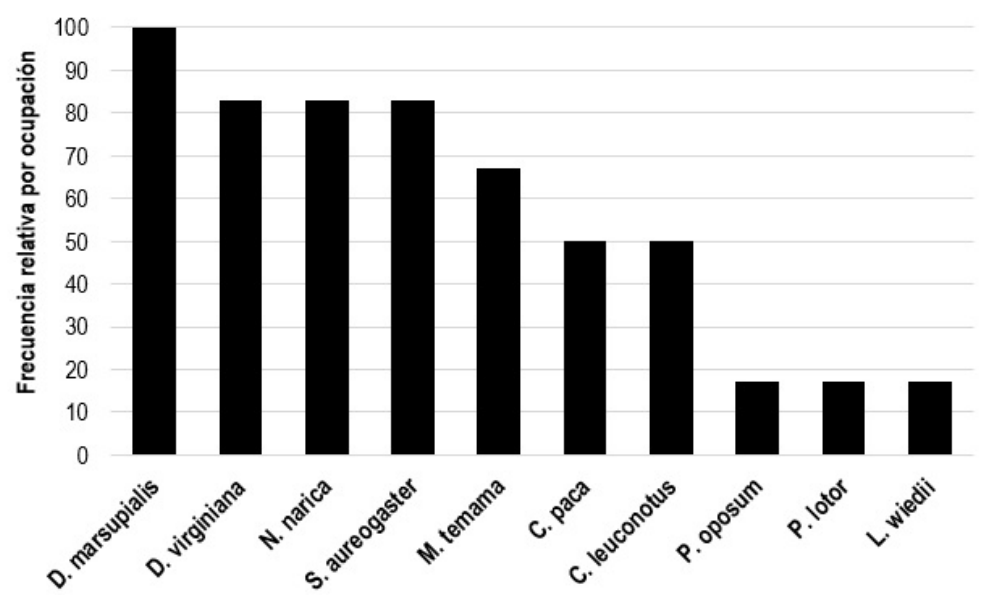

Figura 4. Frecuencia relativa por ocupación (FRO) de mamíferos del cerro Acontecatl, Zongolica, Veracruz.

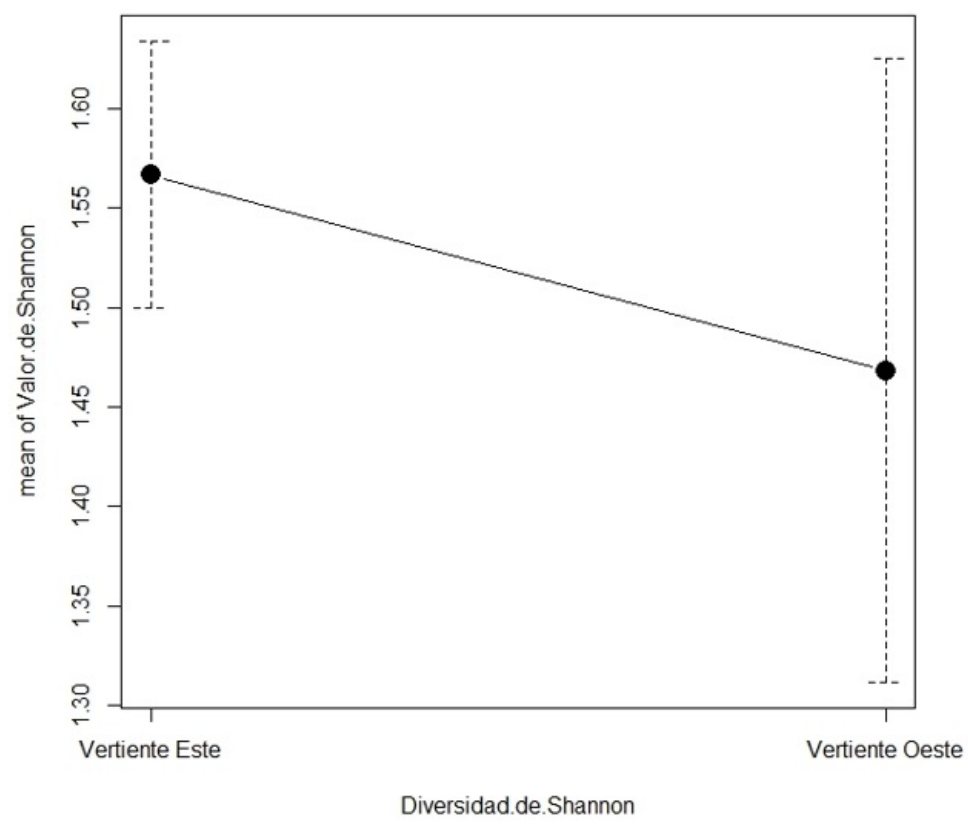

Figura 5. Índice de diversidad de Shannon $\mathrm{H}^{\prime}$ de ambas vertientes muestreadas, con sus respectivos intervalos de confianza y re-muestreados 1000 veces por Bootstrap.

diada tiene una importancia ecológica por la diversidad mastofaunística que alberga y que supera a la riqueza que se ha reportado en otras zonas del país (Lira-Torres y Briones-Salas 2012, Cruz-Jácome et al. 2015). Las 11 especies registradas de las 27 con distribución potencial en la región, puede indicar que las otras 16 especies de mamíferos no se encuentren actualmente en el área de estudio, posible- mente porque el ecosistema no reúne las características biológicas básicas que requieren las especies, o que simplemente han desaparecido de la región (González-Christen y Delfín-Alfonso 2016).

Las mayores abundancias las tuvo la especie $N$. narica, debido a su estructura social, pues puede alcanzar grupos de hasta 20 individuos, sumando también su alta tasa de reproductiva; en tanto que 

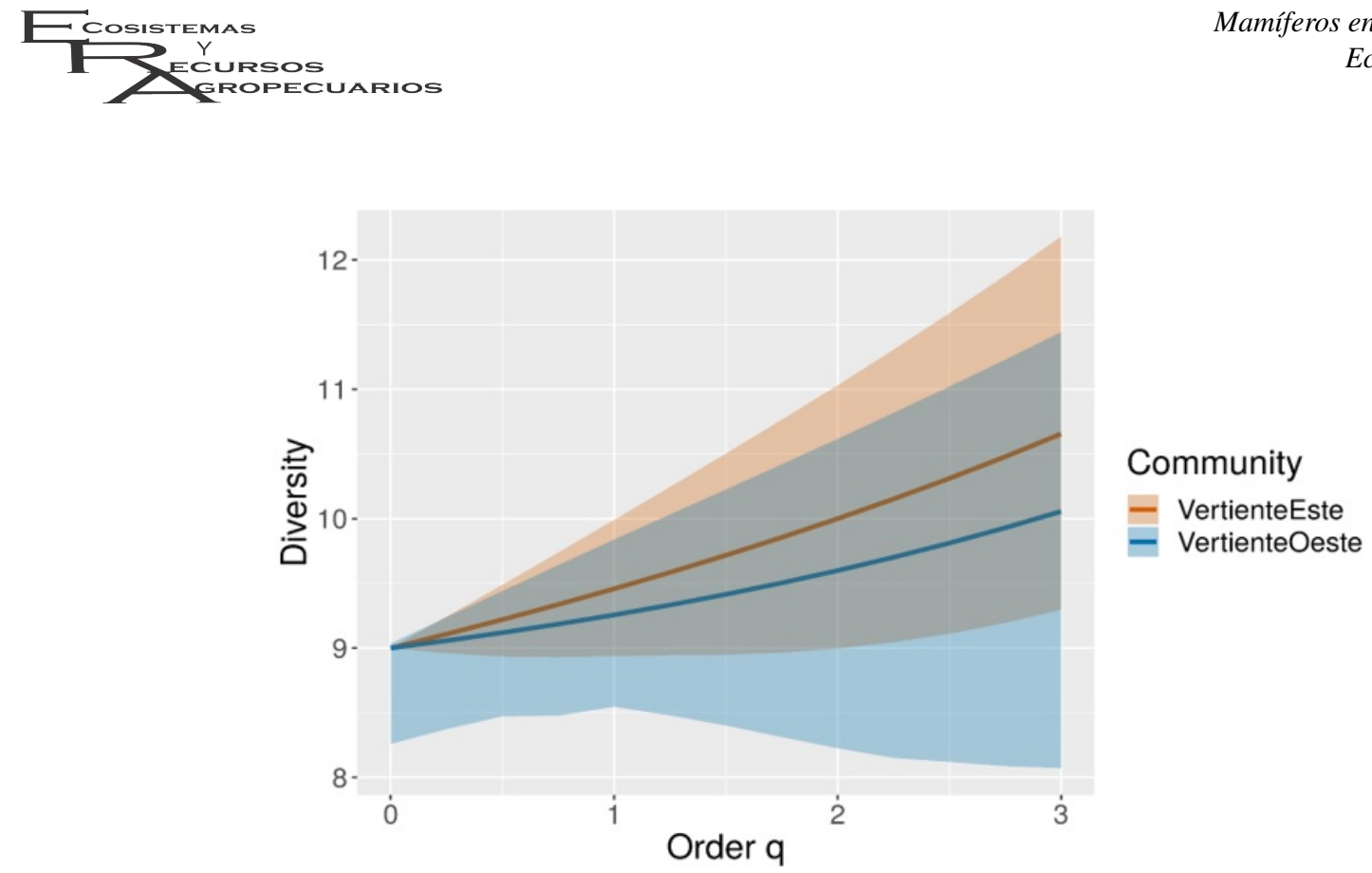

Figura 6. Curva de riqueza de mamíferos de ambas vertientes muestreadas, con sus respectivos intervalos de confianza y re-muestreados 100 veces por Bootstrap.

D. marsupialis tuvo el mayor registro, ya que estuvo presente en el $100 \%$ de los registros de fototrampas y transectos, lo que se podría deber a su adaptabilidad a diferentes ambientes (Ceballos y Oliva 2005). Mientras que L. wiedii y P. oposum tuvieron una baja frecuencia relativa de ocurrencia $(<$ $1 \%)$, esto probablemente puede ser explicado por su alta sensibilidad a la presencia humana, así como a las actividades de cacería furtiva detectadas en la zona de estudio y que merman las poblaciones de mamíferos (Lira-Torres y Briones-Salas 2011). Con base en Villareal et al. (2006) y al índice de Margalef obtenido, se considera que el Cerro Acontecatl, tiene una diversidad de mamíferos media (Margalef 1972) en cuanto a riqueza y abundancia de especies de mamíferos. Esto permite deducir que, pese a la perturbación del área de estudio, si se realizan acciones de conservación, las poblaciones de los mamíferos presentes pueden protegerse a corto plazo, pero si se incrementa la cobertura forestal y se conecta mediante corredores biológicos con otros remanentes de vegetación (Krausman 2002), la conservación de estas y otras poblaciones de fauna silvestre, sería efectiva a largo plazo (Pina et al. 2004).

No se observaron diferencias significativas en la riqueza y abundancia de mamíferos en la vertiente
Oeste y Este, debido a la similitud en la abundancia y riqueza registrada en ambos sitios, probablemente esto sea resultado de los desplazamientos y movimiento que las especies de mamíferos hacen como parte de su actividad biológica en la búsqueda de alimento o refugio (Pérez-Irineo y Santos-Moreno 2010). En este sentido, el Cerro Acontecatl contiene un remanente de BMM que otorga recursos básicos para el mantenimiento de las poblaciones de los mamíferos registrados, manteniendo un equilibrio en el ensamblaje de la comunidad de mamíferos (PérezIrineo y Santos-Moreno 2012). Por lo que se requieren considerar acciones para fomentar los estudios sobre la biodiversidad que habita en el Cerro Acontecatl. Es imprescindible disminuir la tasa de deforestación de la región y conectar los remantes de vegetación con otros sistemas de conservación como las Áreas Naturales Protegidas, lo cual permitiría que las especies de mamíferos puedan vivir en la región (Rodríguez-Macedo et al. 2014).

Las actividades antropogénicas como la cacería y fragmentación del hábitat que se observa en la zona de estudio posiblemente estén afectando de manera indirecta a la riqueza y abundancia de mamíferos del Cerro Acontecatl, ya que en las fotocapturas se encontró evidencia de perros de cacería 
minutos después de la fotocaptura de $M$. temama, así como fotos de personas realizando extracción de madera. Es pertinente concientizar a la sociedad de la sierra de Zongolica sobre la importancia en la conservación de la biodiversidad, para mantener el equilibrio entre sociedad-fauna silvestre (Fuller et al. 2006).

\section{CONCLUSIONES}

Se reporta el primer listado de mamíferos medianos y grandes del Cerro Acontecatl, municipio de Zongolica Veracruz. Se registraron 11 especies de mamíferos mediante fototrampeo y transectos, y un total de 16 especies de mamíferos reportados por la literatura con distribución potencial en la región. Las especies $L$. wiedii y $L$. pardalis pueden conservarse como especies sombrilla, con lo que se protegería su hábitat y otras especies de fauna silvestre asociadas. La diversidad de mamíferos registrada es indicadora de un ecosistema medianamente rico en especies y abundancias, pero la actividad antropogénica en la zona interfiere con el equilibrio del ecosistema.

\section{AGRADECIMIENTOS}

Al Colegio de Postgraduados, Campus Córdoba, por el financiamiento otorgado para el trabajo de campo y por el equipo de fototrampeo. Al proyecto Caracterización de recursos zoogenéticos de las altas montañas, Veracruz: aplicación de la filogeografía y modelación ecológica (PRODEP: 5116/18-9245/PTC-896) por las gestiones para concretar esta publicación. Al Editor asociado y los revisores anónimos que con sus observaciones ayudaron a mejorar este trabajo.

\section{LITERATURA CITADA}

Altamirano-Álvarez TA, Soriano-Sarabia M, García-Bernal AJ, Miranda-González, NP, Jiménez-Gutiérrez, BE (2009) Mamíferos medianos y grandes de la comunidad El Paredón, Miacatlán, Morelos, México. Revista de Zoología 20: 17-29.

Aranda M (2000) Huellas y otros rastros de los mamíferos grandes y medianos de México. Instituto de Ecología. México. 212p.

Aranda M (2012) Manual para el rastreo de mamíferos silvestres de México. Comisión Nacional para el Conocimientos y Uso de la Biodiversidad. México. 255p.

Ávila-Nájera DM, Chávez C, Lazcano-Barrero MA, Mendoza GA, Pérez-Elizalde S (2016) Traslape en patrones de actividad entre grandes felinos y sus principales presas en el norte de Quintana Roo, México. Therya 7: 439-448.

Ceballos G, Arroyo-Cabrales J (2012) Lista actualizada de los mamíferos de México. Revista Mexicana de Mastozoología 9: 21-71.

Ceballos G, Arroyo-Cabrales J, Medellín RA (2002) Mamíferos de México. In: Ceballos G, Simonetti JA (ed.) Diversidad y conservación de los mamíferos neotropicales. CONABIO, UNAM. México. pp: 87-107.

Ceballos G, Brown JH (1995) Global patterns of mammalian diversity, endemism, and endangerment. Conservation Biology 9: 559-568.

Ceballos G, Oliva G (2005) Los mamíferos silvestres de México. Fondo de cultura económica. México. 986p.

Chao A, Jost $L$ (2012) Coverage-based rarefaction and extrapolation: standardizing samples by completeness rather than size. Ecology 93: 2533-2547.

Chao A, Ma KH, Hsieh TC (2016) iNEXT (iNterpolation and EXTrapolation) online: Software for Interpolation and Extrapolation of Species Diversity. Program and User's Guide. Disponible en: http://chao.stat.nthu.edu.tw/ wordpress/software_download/. Consultado el 13 de abril de 2019. 
Chávez C, De La Torre A, Bárcenas H, Medellín RA, Zarza H, Ceballos G (2013) Manual de fototrampeo para estudio de fauna silvestre. El jaguar en México como estudio de caso. Alianza WWF-Telcel, Universidad Nacional Autónoma de México, México. 103p.

Colwell RK (2006) EstimateS: statistical estimation of species richness and shared species from samples. Version 8. User's guide and application published. Disponible en: http://viceroy.eeb.uconn.edu/ estimates. Consultado: el 20 de mayo de 2018.

Colwell RK (2013) EstimateS: Estimación estadística de la riqueza de especies y especies compartidas a partir de muestras. Versión 9. Guía del usuario y aplicación; acceso: http://purl.oclc.org/estimates. Consultado: el 21 de agosto de 2015.

CONABIO (2011) La biodiversidad en Veracruz: Estudio de Estado. Comisión Nacional para el Conocimiento y Uso de la Biodiversidad. Gobierno del Estado de Veracruz, Universidad Veracruzana, Instituto de Ecología. México 545p.

Cruz-Jácome O, López-Tello E, Delfín-Alfonso CA, Mandujano S (2015) Riqueza y abundancia relativa de mamíferos medianos y grandes en una localidad en la Reserva de la Biosfera Tehuacán-Cuicatlán, Oaxaca, México. Therya 6: 45-448.

Fuller T, Munguía M, Mayfield M, Sánchez-Cordero V, Sarkar S (2006) Incorporating connectivity into conservation planning: a multi-criteria case study from central Mexico. Biological Conservation 133: 131-142.

Gallina S, González-Romero A (2018) La conservación de mamíferos medianos en dos reservas ecológicas privadas de Veracruz, México. Revista Mexicana de Biodiversidad 89: 1245-1254.

Gaona S, González-Christen A, López-Wilchis R (2003) Síntesis del conocimiento de los mamíferos silvestres del Estado de Veracruz, México. Revista de la Sociedad Mexicana de Historia Natural 3a época 1: 91-124.

García-Burgos J, Gallina S, González-Romero A (2014) Relación entre la riqueza de mamíferos medianos en cafetales y la heterogeneidad espacial en el centro de Veracruz. Acta Zoológica Mexicana 30: 337-356.

García-Franco JG, Castillo-Campos G, Mehltreter K, Martínez ML, Vázquez G (2008) Estructura y composición de un bosque mesófilo del centro de Veracruz, México. Boletín de la Sociedad Botánica de México 83: 37-52.

González-Christen A (2006) Los mamíferos amenazados de Veracruz. La Ciencia y el Hombre 19: 27-32.

González-Christen A (2010) Los mamíferos de Veracruz. Guía ilustrada. Colección la Ciencia en Veracruz, Consejo Veracruzano de Investigación Científica y Desarrollo Tecnológico. Veracruz, México. 191p.

González-Christen A (2011) Mamíferos: Distribución, endemismo y estado de conservación. In: Cruz-Angón A (ed.) La biodiversidad en Veracruz: Estudio de Estado. Comisión Nacional para el Conocimiento y Uso de la Biodiversidad, Gobierno del Estado de Veracruz, Universidad Veracruzana, Instituto de Ecología. Xalapa, México. pp: 579-592.

González-Christen A, Delfín-Alfonso CA (2016) Los mamíferos terrestres de Veracruz, México y su protección. En: Briones-Salas M, Hortelano-Moncada Y, Magaña-Cota G, Sánchez-Rojas G, Sosa-Escalante JE (eds.) Riqueza y conservación de los mamíferos en México a nivel estatal. Instituto de Biología, Universidad Nacional Autónoma de México, Asociación Mexicana de Mastozoología, Universidad de Guanajuato. Ciudad de México, México. pp: 499-534.

González-Ruiz N, Ramírez-Pulido J, Gual-Díaz M (2014) Mamíferos del bosque mesófilo de montaña en México. En: Gual-Díaz M, Rendón-Correa A (Eds.). Bosques mesófilos de montaña de México: diversidad, ecología 
y manejo. Comisión Nacional para el Conocimiento y Uso de la Biodiversidad. Ciudad de México, México. pp: 305-326.

Hernández-Baz F, Rodríguez-Vargas DU (2014) Libro rojo de la fauna del estado de Veracruz. Gobierno del Estado de Veracruz, Procuraduría Estatal de Protección al Medio Ambiente, Universidad Veracruzana. México. 220p.

Hernández-Huerta A (1994) Instituciones que estudian y trabajan en la protección de la Fauna de Veracruz. In: González-Christen A, González-Romero A (eds.) Problemática Ambiental en el Estado de Veracruz: Recursos Faunísticos. Colegio Profesional de Biólogos del Estado de Veracruz, Gobierno del Estado de Veracruz, Universidad Veracruzana. Veracruz, México. pp: 81-90.

Krausman P (2002) Introduction to wildlife management: the basics. Prentice Hall, Nueva Jersey, EEUU. 478p.

Lavariega MC, Briones-Salas M, Gómez-Ugalde RM (2012) Mamíferos medianos y grandes de la Sierra de ViIla Alta, Oaxaca, México. Mastozoología Neotropical 19: 225-241

Lira-Torres I, Briones-Salas M (2011) Impacto de la ganadería extensiva y cacería de subsistencia sobre la abundancia relativa de mamíferos en la Selva Zoque, Oaxaca, México. Therya 2: 217-244.

Lira-Torres I, Briones-Salas M (2012) Abundancia relativa y patrones de actividad de los mamíferos de los Chimalapas, Oaxaca, México. Acta Zoológica Mexicana 28: 566-585.

Mandujano RS (2011) Ecología de poblaciones aplicada al manejo de fauna silvestre: Cuatro conceptos ( $N, \lambda$, MSY, PE). Colección manejo de fauna silvestre. Instituto Literario de Veracruz. Veracruz, México. 102p.

Margalef R (1972) Homage to E. Hutchison, or why is there an upper limit to diversity. Transactions of the Connecticut Academy of Arts and Sciences 44: 211-235

Moreno C (2001) Métodos para medir la biodiversidad. M\&T Manuales y Tesis. Sociedad Entomológica Aragonesa (SEA). Zaragoza, España. 84p.

Moreno CE, Barragán F, Pineda E, Pavón NP (2011) Reanalyzing alpha diversity: alternatives to understand and compare information about ecological communities. Revista Mexicana de Biodiversidad 82: 1249-1261.

Pérez-Irineo G, Santos-Moreno A (2010) Diversidad de una comunidad de mamíferos carnívoros en una selva mediana del noreste de Oaxaca, México. Acta Zoológica Mexicana 26: 721-736.

Pérez-Irineo G, Santos-Moreno A (2012) Diversidad de mamíferos terrestres de talla grande y media de una selva subcaducifolia del noreste de Oaxaca, México. Revista Mexicana de Biodiversidad 83: 164-169.

Pina GPL, Gómez RAC, González CAL (2004) Distribution, habitat association and activity patterns of medium and large sized mammals of Sonora, Mexico. Natural Areas Journal 24: 354-357.

Ramírez-Pulido J, Arroyo-Cabrales J, Castro-Campillo A (2005) Estado actual y relación nomenclatural de los mamíferos terrestres de México. Acta Zoológica Mexicana 21: 21-82.

Ramírez-Pulido J, González-Ruiz N, Gardner AL, Arroyo-Cabrales J (2014) List of recent land mammals of Mexico, 2014. Natural Science Research Laboratory. Museum of Texas Tech University. Especial Publications. USA. 69p.

Rodríguez-Macedo M, González-Christen A, León-Paniagua LS (2014) Diversidad de los mamíferos silvestres de Misantla, Veracruz, México. Revista Mexicana de Biodiversidad 85: 262-275.

SEMARNAT (2010) Norma Oficial Mexicana NOM-059-SEMARNAT-2010, Protección Ambiental-Especies nativas de México de Flora y Fauna Silvestres-Categorías de Riesgo y Especificaciones para su inclusión, 
exclusión o cambio-Lista de especies en riesgo. Diario Oficial de la Federación \#56, 2a Sección. México. $85 \mathrm{p}$.

Stoner KE, Riba-Hernández P, Vulinec K, Lambert JE (2007) The role of mammals in creating and modifying seedshadows in tropical forests and some possible consequences of their elimination. Biotropica 39: 316327.

Tirira D (1998) Técnicas de campo para el estudio de mamíferos silvestres. En: Tirira D (Ed.). Biología, sistemática y conservación de los mamíferos del Ecuador. Museo de Zoología, Centro de Biodiversidad y Ambiente. Pontifica Universidad Católica del Ecuador. Quito, Ecuador. pp: 93-126.

Tlapaya L, Gallina S (2010) Cacería de mamíferos medianos en cafetales del centro de Veracruz, México. Acta Zoológica Mexicana 26: 259-277.

Torres-Romero EJ, Espinoza-Medinilla E, Lazcano-Barrero MA, Maffei L (2017) Ecology and conservation of ocelot (Leopardus pardalis) in Northern Quintana Roo, Mexico. Therya 8: 11-18.

Villareal H, Álvarez M, Córdoba S, Escobar F, Agua GF, Gast F, et al. (2006) Manual de métodos para el desarrollo de inventarios de biodiversidad. Programa de Inventarios de Biodiversidad. Instituto de Investigaciones de Recursos Biológicos Alexander Von Humboldt. Bogotá, Colombia. 236p.

Wilson DE, Reeder DM (2005) Mammals species of the world; a taxonomic and geographic reference. Johns Hopkins University Press, Baltimore, EU. 2142p.

Wilson DE, Reeder DM (2011) Class Mammalia Linnaeus, 1758. In: Zhang ZQ (ed.). Animal biodiversity: An outline of higher-level classification and survey of taxonomic richness. Zootaxa 3148: 56-60. 